\section{Deep sclerectomy in pediatric glaucoma filtering surgery}

\author{
Abstract \\ Purpose To study the additive value of deep \\ sclerectomy to the procedure of combined \\ trabeculotomy-trabeculectomy with \\ mitomycin C (CTTM) for the treatment of \\ primary congenital glaucoma. \\ Study design This study is a prospective, \\ randomized case series. \\ Patients and methods The study was \\ conducted on 20 eyes of 20 children with \\ primary congenital glaucoma presenting to \\ the Department of Ophthalmology of the \\ Alexandria Main University Hospital. \\ Preoperative examination under anesthesia \\ was followed by surgical intervention. \\ Postoperative examinations were conducted \\ immediately after surgery and at 1, 2, 3, 6, 9, \\ and 12 months. Intraoperative and \\ postoperative complications, as well as \\ operative time, were recorded. \\ Results The mean ( $\pm S D$, range) age of the \\ study patients in the CTTM group and in the \\ combined trabeculotomy-trabeculectomy \\ with mitomycin $C$ with deep sclerectomy \\ (CTTM-DS) group was $4.7( \pm 2.0,2-8)$ and 7.0 \\ ( $\pm 3.8,3-13)$ months, respectively. The mean \\ ( $\pm S D$, range) preoperative intraocular \\ pressure (IOP) in the CTTM and CTTM-DS \\ groups was $16.7(4.3,10-26)$ and $16.4(8.4$, \\ 8-36), respectively, and these dropped at 12 \\ months of follow-up to $4.9(2.0,2-8)$ and 5.6 \\ $(3.3,2-10)$, respectively. The mean $( \pm S D$, \\ range) of the duration of the operation in the \\ CTTM and the CTTM-DS was $57( \pm 8,50$ \\ $71) \mathrm{min}$ and $53( \pm 7,42-64) \mathrm{min}$, respectively \\ $(P=0.428)$. Two eyes $(20 \%)$ in the CTTM-DS \\ group developed hypotony disc edema at the \\ first 2 months and resolved spontaneously \\ thereafter. No other complications were noted \\ in either of the groups. \\ Conclusion The addition of deep \\ sclerectomy to the procedure of CTTM in \\ pediatric glaucoma surgery facilitates the \\ finding of Schlemm's canal, shortens the \\ duration of surgery, and is not associated
}

with any additional complications. Hence, the author recommends the addition of deep sclerectomy to CTTM surgery for primary congenital glaucoma.

Eye (2012) 26, 1548-1553; doi:10.1038/eye.2012.215; published online 12 October 2012

Keywords: pediatric; glaucoma; deep; sclerectomy; trabeculotomy; trabeculectomy

Introduction

Pediatric glaucoma is generally a surgical disease ${ }^{1}$ with medical treatment being used only as a temporary measure or in preparation for surgery. ${ }^{2,3}$ Primary congenital glaucoma is the most common of the childhood glaucomas. ${ }^{4}$ A variety of surgical procedures have been used for the treatment of primary congenital glaucoma, including goniotomy (for cases of clear cornea), ${ }^{5}$ trabeculotomy, ${ }^{6}$ trabeculectomy, ${ }^{3,7,8}$ and glaucoma drainage devices. ${ }^{8}$ The use of wound-modulating agents, including antimetabolites such as 5-flourouracil and mitomycin $\mathrm{C}$, has added to the success of filtering surgery in pediatric glaucoma. ${ }^{3,7,9}$ Of particular interest, lately the procedures for primary congenital glaucoma are combined, ${ }^{10,11}$ especially trabeculotomy-trabeculectomy with mitomycin C, with variably reported success rates. ${ }^{1,11,12}$

Childhood glaucoma is more common and more severe in communities with higher consanguineous marriages, such as in Romanian Gypsies and in the Saudi Arabian population. ${ }^{11,13}$ In these communities, the disease is more severe and combined procedures are more successful in controlling the disease. ${ }^{11}$

The performance of an ab-externo trabeculotomy involves the identification of the Schlemm's canal from an ab-externo approach and introducing a trabeculotome in it; this can be quite challenging, given the distorted limbal anatomy commonly encountered in cases of 
primary congenital glaucoma. However, the dissection of a deep scleral flap allows the direct visualization of the scleral spur, as well as the direct opening and deroofing of Schlemm's canal, ${ }^{14}$ thus rendering the identification of Schlemm's canal a relatively easier task.

In this study, the author prospectively studied the additive value of a deep sclerectomy to the standard procedure of combined trabeculotomy-trabeculectomy with mitomycin C (CTTM) for the surgical treatment of primary congenital glaucoma cases.

\section{Subjects and methods}

This is a prospective comparative study aiming at the evaluation of the additive value of deep sclerectomy to CTTM in pediatric glaucoma surgery. The study was conducted on 20 eyes of 20 children presenting with the diagnosis of primary congenital glaucoma and operated upon in the Department of Ophthalmology of the Alexandria Main University Hospital. The study was granted approval by the ethics committee of the Faculty of Medicine of Alexandria University. Informed consent was obtained from the parents and care givers of all children participating in the study after explanation of the risks and benefits of the procedure.

After taking a thorough history from the parents or care providers of the child, an initial office examination was conducted and was followed by an examination under general anesthesia (GA). Inhalational sevoflorane (fluoromethyl hexafluoroisopropyl ether) anesthesia was used for all children. Examination of children with glaucoma follows a standard protocol in the Alexandria Main University Hospital. All examinations were carried out by the same ophthalmologist (the author). The author had previously described a similar protocol for examination of children with congenital cataract at the Alexandria Main University Hospital. ${ }^{15}$ Examination of children under GA in the Alexandria Main University Hospital is conducted in a different setting from the surgical suite, ie examination is conducted at a different date from the date of the surgical procedure. Measurement of intraocular pressure (IOP) was conducted using applanation tonometry (Perkin's tonometer, Haag Streit USA and Reliance Medical Products, Mason, OH, USA) just after the induction of anesthesia and with the eyes in the central position and without intubation of the child. Owing to the known reduction of IOP induced by inhalational GA, if the measured IOP was low enough to preclude the diagnosis of primary congenital glaucoma, the diagnosis was then based on serial follow-up of the suspected eyes, with the documentation of progression of the optic nerve cupping, corneal diameter, and axial length being a prerequisite for the establishment of the diagnosis, rather than the measured absolute value of the IOP. The patient was then scheduled for surgery. A CTTM was conducted in all the patients. Intraoperatively, eyes were randomized for the procedure of CTTM alone or with the addition of a deep sclerectomy. Randomization was carried out by a flip coin choice by an attending assistant nurse.

Randomization was not carried out preoperatively in order to avoid surgeon bias in changing the thickness of the scleral flap created during the initial part of the surgery, tending to make it thinner in cases in which deep sclerectomy was planned and thicker (deeper dissection) in cases without deep sclerectomy.

The combined procedure involves a corneal traction suture superotemporally and an inferior paracentesis. This is followed by a conjunctival peritomy in the superotemporal quadrant and localized tenonectomy. A triangular scleral flap was then fashioned $4 \times 4 \times 4 \mathrm{~mm}$, half the scleral thickness, and dissection was carried forward for $2 \mathrm{~mm}$ into clear cornea, followed by the application of mitomycin C, $0.4 \mathrm{mg} / \mathrm{ml}$ for $2 \mathrm{~min}$ and then thorough irrigation. At this step randomization was carried out. For eyes randomized for no deep sclerectomy, radial incisions in the bed of the flap in the region of the scleral spur were made to identify Schlemm's canal, and a trabeculotomy was performed (see Supplementary Video 1). For eyes randomized for deep sclerectomy, a deep scleral flap was marked, $2 \times 2 \mathrm{~mm}$, and dissection was carried forward until deroofing of Schlemm's canal and then a trabeculotomy was performed (see Supplementary Video 2). The Thomas Neuhann U-shaped trabeculotome with no handle (catalog product number G-15138, from Geuder Instruments, Heidelberg, Germany) was used to perform the trabeculotomy. The entire length of the trabeculotome was introduced into Schlemm's canal and used to sweep into the anterior chamber, nasally and temporally, thus treating an average of 70 degrees of the angle. The combined procedure was then completed by a trabeculectomy by Kelly Descemet punch, a peripheral iridectomy, and then closure by 10/0 nylon sutures of the sclera flap and the overlying conjunctiva. The procedure was completed by the subconjunctival injection of a depot steroid (betamethasone). The total duration of the procedure was documented for all cases.

Postoperative treatment was standard. Antibiotic (ofloxacin) and steroid (dexamethasone) eye drops were used hourly, with a cycloplegic (cyclopentolate) used three times a day in the early postoperative period and up to 1 week, followed by gradual tapering of the steroids over a lengthy period of 6 weeks, with reduction of antibiotic dose and cessation of the cycloplegic.

Patients were examined immediately after surgery and at 1, 2, 3, 6, 9, and 12 months. Follow-up examinations were conducted in the same scheme as the preoperative examinations. 
Success was defined as an IOP of $<16 \mathrm{mmHg}^{16}$ under GA with no hypotony complications and/or no progression of the disease as evidenced by any progression of the ocular parameters as the corneal diameter, cup/disc ratio and axial length beyond the normal growth curves of the eye for that age group. The definition of success did not include a lower limit for the IOP, as in many cases in which the measured IOP under GA was apparently very low $(<6 \mathrm{mmHg})$ the child would demonstrate relatively normal visual behavior (fixation and following), as well as no resistance to covering either eye, and the parents would describe a normal visual pattern for the child, similar to that of healthy siblings. Hence, such a low IOP was not classified as failure. In addition, the effect of sevoflurane on lowering the IOP measured under GA is well documented $^{17}$.

Data were collected, tabulated, and statistically analyzed. Data were analyzed using the Statistical Package for Social Sciences (SPSS version 17, IBM, Chicago, IL, USA).

The distributions of quantitative variables were tested for normality using the Kolmogorov-Smirnov test, which revealed normal distribution of some data and abnormal distribution of others. Thus, nonparametric statistics were applied. Quantitative data were described using median, minimum, and maximum. Qualitative data were described using number and percent. Comparison of two quantitative dependent variables repeated over time (at onset and after follow-up) were conducted using the Friedman test. Comparison of quantitative variables between the two groups was conducted using the Mann-Whitney U-test. In all statistical tests, a level of significance of 0.05 was used, below which the results were considered to be statistically significant.

\section{Results}

The study was conducted on 20 eyes of 20 children with the diagnosis of primary congenital glaucoma presenting to and operated upon in the Department of Ophthalmology of the Alexandria Main University Hospital. The demographic data of the patients are presented in Table 1. The clinical examination data and the axial length measurements for the study patients are presented in Table 2. There were no statistically significant differences between the two groups of patients as regards the preoperative variables, including IOP, corneal diameter, cup/disc ratio, and axial length (the $P$-values for the age at surgery and the axial length were 0.155 and 0.233 , respectively, between the two groups), as well as along all the follow-up periods until the end of follow-up in both the groups. There was an
Table 1 Patient demographics and operations durations

\begin{tabular}{|c|c|c|}
\hline \multicolumn{3}{|c|}{ Patient demographics and operations durations } \\
\hline & CTTM & CTTM-DS \\
\hline Age in months & $4.7 \pm 2.0$ & $7.0 \pm 3.8$ \\
\hline$($ mean $\pm \mathrm{SD}$, (median, range) $)$ & $(4,2-8)$ & $(5,3-13)$ \\
\hline \multicolumn{3}{|l|}{ Gender (n, \%) } \\
\hline Male & $6(60 \%)$ & $8(80 \%)$ \\
\hline Female & $4(40 \%)$ & $2(20 \%)$ \\
\hline \multicolumn{3}{|l|}{ Laterality (n, \%) } \\
\hline Right & $8(80 \%)$ & $5(50 \%)$ \\
\hline Left & $2(20 \%)$ & $5(50 \%)$ \\
\hline $\begin{array}{l}\text { Duration of follow-up in } \\
\text { months (mean } \pm S D \text {, (range)) }\end{array}$ & $18.5 \pm 9.2(8-35)$ & $14.6 \pm 4.3(6-20)$ \\
\hline $\begin{array}{l}\text { Duration of operation in } \\
\text { minutes (mean } \pm S D \text {, (range)) }\end{array}$ & $57 \pm 8(50-71)$ & $53 \pm 7(42-64)$ \\
\hline
\end{tabular}

Abbreviations: CTTM, combined trabeculotomy-trabeculectomy with mitomycin C; CTTM-DS, combined trabeculotomy-trabeculectomy with mitomycin $\mathrm{C}$ with deep sclerectomy.

apparently lower IOP in the second month in the combined trabeculotomy-trabeculectomy with mitomycin C with deep sclerectomy (CTTM-DS) group than the group without deep sclerectomy, although not statistically significant $(P=0.056)$. The blebs in both the groups were alike: pale, diffuse, and low. No bleb leak was detected at any follow-up time period. The percentage reductions of IOP in either group at different follow-up time points are demonstrated in Table 3 . The average $( \pm S D$, range) of the duration of the operation in the CTTM and the CTTM-DS was $57( \pm 8,50-71) \mathrm{min}$ and $53( \pm 7,42-64) \mathrm{min}$, respectively. There was no statistically significant difference between the two groups of patients as regards the duration of the operation $(P=0.428)$. Success of every procedure was judged at every follow-up time point and was $100 \%$ for both procedures at every follow-up time point except for the two eyes in the CTTM-DS group that showed mild hypotony disc edema only at the 1-month and 2-month follow-up visits and that resolved spontaneously by the 3rd month follow-up visit despite the persistence of a measured low IOP. None of the eyes in either group showed hypotony maculopathy. Despite the measured low IOP in the study eyes, the children demonstrated normal visual behavior (fixation and following) and no resistance to covering either eyes, and the parents and care givers of the children reported normal visual behavior, similar to that of healthy siblings. A negative significant correlation was found between the age at surgery and the operative duration in the CTTM group (correlation coefficient $=-0.882, P=0.020$ ). No correlation was found between any of the postoperative variables at any time point of follow-up and any of the 


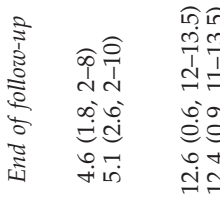

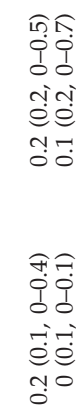

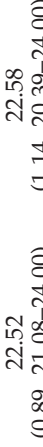

लె

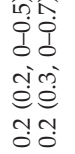$$
\text { ำ }
$$

preoperative variables or the patient characteristics in either group.

\section{Discussion}

In this study, CTTM was used in the surgical treatment of 20 eyes with primary congenital glaucoma. The follow-up was continued for more than 1 year. All cases were successful in terms of IOP control, stability of ocular parameters (corneal diameter, optic nerve cupping, and axial length) and the absence of significant or vision-threatening complications. Comparable results were reported by studies conducted on similar patient populations, such as the report by Mullaney et $a l^{12}$ who achieved $78 \%$ success rates with CTTM in Saudi Arabia, Elder ${ }^{1}$ who achieved a success rate of $93.5 \%$ in Israel with combined trabeculotomytrabeculectomy without mitomycin C, and Mandal et $a l^{18}$ who achieved a success rate of $94.4 \%$ in India, again with combined trabeculotomy-trabeculectomy without mitomycin C. The results of the present study are clearly much better than the report by Al Hazmi et $a l^{19}$ in 1998, where the achieved success rate was as low as $57 \%$ for cases of primary congenital glaucoma using the CTTM procedure, probably related to the discrepancy in sample size, with a fewer number of cases in the present study.

In the present study, deep sclerectomy was added to CTTM and was fully successful at all time points and at the end of the follow-up. There are several reports in the literature about the use of non-penetrating deep sclerectomy and the use of combined deep sclerectomy and trabeculectomy (penetrating deep sclerectomy) in pediatric glaucoma. Denis et al ${ }^{16}$ reported a total success of $56 \%$ and a partial success of $89 \%$ using deep sclerectomy with 5-flourouracil application for early diagnosed primary congenital glaucoma. Roche et $a l^{20}$ reported a success rate of $93 \%$ with deep sclerectomy in primary congenital glaucoma, although occasionally with multiple surgeries and with IOPlowering medications. Feusier et al ${ }^{21}$ reported a complete and qualified success rate of $52.3 \%$ and $70.6 \%$, respectively, with the use of penetrating deep sclerectomy (combined deep sclerectomy and trabeculectomy). To our knowledge, there are no published data about the combination of deep sclerectomy and CTTM.

In the present study, the addition of deep sclerectomy was not associated with any additional complications, except for a mild incidence of hypotony in the early postoperative period, which was self-limiting. This study also demonstrated the interesting finding that in children's eyes hypotony seemed to be relatively well tolerated than in adults, with little-if any-effect on 
Table 3 Percentage reduction of IOP for CTTM and CTTM-DS patients at time points of follow-up in months

\begin{tabular}{|c|c|c|c|c|c|c|c|}
\hline \multicolumn{8}{|c|}{ Percentage reduction of IOP for CTTM and CTTM-DS patients } \\
\hline Mean (SD) & 1 & 2 & 3 & 6 & 9 & 12 & $\begin{array}{c}\text { End of } \\
\text { follow-up }\end{array}$ \\
\hline \multicolumn{8}{|l|}{ IOP $(m m H g)$} \\
\hline СТTM & $64(28)$ & $52(24)$ & $67(18)$ & $63(24)$ & 77 (14) & $69(16)$ & 72 (12) \\
\hline CTTM-DS & $55(42)$ & $64(23)$ & $52(18)$ & $62(22)$ & $66(20)$ & $62(36)$ & $63(26)$ \\
\hline
\end{tabular}

Abbreviations: CTTM, combined trabeculotomy-trabeculectomy with mitomycin C; CTTM-DS, combined trabeculotomy-trabeculectomy with mitomycin $\mathrm{C}$ with deep sclerectomy.

visual function. Another explanation could be the IOP-lowering effect of inhalational sevoflurane anesthesia, ${ }^{17}$ in which case the actual IOP would be higher than the measured IOP, and this might explain the apparently normal visual function of these apparently hypotonous eyes. The addition of deep sclerectomy also resulted in a shortening of the total duration of the operative procedure (although not to a statistically significant level) and hence less postoperative inflammation and less exposure of the child to the GA with its antecedent risks. Although not encountered in the present study, the inability to identify Schlemm's canal and hence failure to perform a trabeculotomy, ${ }^{1}$ as well as accidental perforation into the anterior chamber during the search for Schlemm's canal, are known incidents in pediatric glaucoma surgery. Hence, the author suggests the addition of deep sclerectomy as a greater guarantee to finding the canal of Schlemm and ensuring the performance of a trabeculotomy; in fact, even if a filtering surgery was not planned and it was the surgeon's intent to perform a trabeculotomy only, or if it was the surgeon's intent to perform a combined trabeculotomy-trabeculectomy without mitomycin C, a deep sclerectomy would help identify Schlemm's canal.

In the present study, patient's age was a determining factor for the duration of surgery in the CTTM procedure. Indeed, the younger the child, the smaller the eye and the palpebral fissure, the more distorted the anatomy and the thinner the sclera; all these factors prolong the surgical procedure. This is somehow compensated (ie, operative duration shortened) by the addition of deep sclerectomy through facilitation of the finding of Schlemm's canal.

A limitation of this study is the relatively small number of patients. Indeed, larger patient series would validate the results more. Another limitation of the study is the apparently different mean age of the two study groups, with the CTTM group patients being younger than the CTTM-DS group patients, yet the age difference is minimal. It is obviously difficult to standardize the age in such a randomized study.
Hence, in conclusion, the use of CTTM is a very useful procedure for the management of primary congenital glaucoma. The addition of deep sclerectomy to the surgical procedure facilitates the finding of Schlemm's canal, shortens the duration of surgery and is not associated with any additional complications. Hence, the author recommends the addition of deep sclerectomy to combined trabeculotomy-trabeculectomy (with or without mitomycin C) surgery for primary congenital glaucoma.

\section{Summary}

What was known before

- Primary congenital glaucoma is the most common form of congenital glaucoma.

- Treatment of primary congenital glaucoma is primarily surgical.

- CTTM provides a better surgical option for difficult and severe cases of primary congenital glaucoma.

- Finding of Schlemm's canal intraoperatively may be difficult owing to distorted limbal anatomy.

What this study adds

- Deep sclerectomy can be added to the procedure of CTTM with no added surgical time, difficulty or complications.

- Adding deep sclerectomy to CTTM facilitates finding Schlemm's canal, hence ensuring performance of the 'trabecultomy' step of CTTM surgery.

\section{Conflict of interest}

The author declares no conflict of interest.

\section{Acknowledgements}

The study was conducted in the Department of Ophthalmology of Alexandria Main University Hospital, Alexandria Faculty of Medicine, Alexandria University, Egypt. There is no prior publication by any other journal. The study was not presented at any meeting or conference. 


\section{References}

1 Elder MJ. Combined trabeculotomy-trabeculectomy compared with primary trabeculectomy for congenital glaucoma. Br J Ophthalmol 1994; 78: 745-748.

2 Turach ME, Aktan G, Idil A. Medical and surgical aspects of congenital glaucoma. Acta Ophthalmol (Scand) 1995; 73: 261-263.

3 Ehrlich R, Snir M, Lusky M, Weinberger D, Friling R, Gaton DD. Augmented trabeculectomy in paediatric glaucoma. $\mathrm{Br}$ J Ophthalmol 2005; 89: 165-168.

4 Taylor RH, Ainsworth JR, Evans AR, Levin AV. The epidemiology of pediatric glaucoma: the Toronto experience. J AAPOS 1999; 3: 308-315.

5 Russell-Eggitt IM, Rice NS, Jay B, Wyse RK. Relapse following goniotomy for congenital glaucoma due to trabecular dysgenesis. Eye 1992; 6: 197-200.

6 Walton DS. Glaucoma in infants and children. In: Nelson LB, Calhoun JH, Harley RD (eds). Pediatric Ophthalmology. W.B. Saunders Company: Philadelphia (PA), 1983, p 588.

7 Rodrigues AM, Paranhos A Jr, Montezano FT, Melo PA, Prata Jr J. Comparison between results of trabeculectomy in primary congenital glaucoma with and without the use of mitomycin C. J Glaucoma 2004; 13: 228-232.

8 Beck AD, Freedman S, Kammer J, Jin J. Aqueous Shunt devices compared with trabeculectomy with mitomycin-C for children in the first two years of life. Am J Ophthalmol 2003; 136: 994-1000.

9 Low S, Hamada S, Nischal KK. Antimetabolite and releasable suture augmented filtration surgery in refractory pediatric glaucomas. J AAPOS 2008; 12: 166-172.

10 Mandal AK, Gothwal VK, Nutheti R. Surgical outcome of primary developmental glaucoma: a single surgeon's longterm experience from a tertiary eye care centre in India. Eye 2007; 21(6): 764-774.

11 Al-Hazmi A, Awad A, Zwaan J, Al-Mesfer SA, Al-Jadaan I, Al-Mohammed A. Correlation between surgical success rate and severity of congenital glaucoma. Br J Ophthalmol 2005; 89: 449-453.

12 Mullaney PB, Selleck C, Al-Awad A, Al-Mesfer S, Zwaan J. Combined trabeculotomy and trabeculectomy as an initial procedure in uncomplicated congenital glaucoma. Arch Ophthalmol 1999; 117: 457-460.

13 Debnath SC, Teichmann KD, Salamah K. Trabeculectomy versus trabeculotomy in congenital glaucoma. $\mathrm{Br} J$ Ophthalmol 1989; 73: 608-611.

14 Mendrinos E, Mermoud A, Shaarawy T. Nonpenetrating glaucoma surgery. Surv Ophthalmol 2008; 53: 592-630.

15 El Shakankiri NM, Bayoumi NH, Abdallah AH, El Sahn MMF. Bottom of formrole of ultrasound and biomicroscopy in evaluation of anterior segment anatomy in congenital and developmental cataract cases. J Cataract Refract Surg 2009; 35: 1893-1905.

16 Denis D, Pommier S, Coste R, Fogliarini C, Benso C, Cornand E. Glaucome congénital et sclérectomie profonde: résultats d'une étude sur plus de 3 années. J Fr Ophtalmol 2008; 3: 173-179.

17 Blumberg D, Congdon N, Jampel H, Gilbert D, Elliott R, Rivers $\mathrm{R}$ et al. The effects of sevoflurane and ketamine on intraocular pressure in children during examination under anesthesia. Am J Ophthalmol 2007; 143: 494-499.

18 Mandal AK, Naduvilath TJ, Jayagandan A. Surgical results of combined trabeculotomy-trabeculectomy for developmental glaucoma. Ophthalmology 1998; 105: 974-982.

19 Al-Hazmi A, Zwaan J, Awad A, al-Mesfer S, Mullarey PB, Wheeler DT. Effectiveness and complications of mitomycin $\mathrm{C}$ use during pediatric glaucoma surgery. Ophthalmology 1998; 105: 1915-1920.

20 Roche O, Beby F, Parsa A, Orssaud C, Dufier JL, Parsa CF. Nonpenetrating external trabeculectomy for congenital glaucoma: a retrospective study. Ophthalmology 2007 2007; 114: 1994-1999.

21 Feusier M, Roy S, Mermoud A. Deep sclerectomy combined with trabeculectomy in pediatric glaucoma. Ophthalmology 2009; 116: 30-38.

Supplementary Information accompanies the paper on Eye website (http://www.nature.com/eye) 